hep-th/9509005

\title{
Off-Diagonal Elements of the DeWitt Expansion from the Quantum Mechanical Path Integral
}

\author{
F.A. Dilkes and D.G.C. McKeon* \\ Department of Applied Mathematics \\ University of Western Ontario \\ London, Ontario, N6A 5B7 \\ Canada
}

(31 August 1995)

(Revised 29 September 1995)

\begin{abstract}
The DeWitt expansion of the matrix element $M_{x y}=\left\langle x\left|\exp -\left[\frac{1}{2}(p-A)^{2}+V\right] t\right| y\right\rangle,(p=-i \partial)$ in powers of $t$ can be made in a number of ways. For $x=y$ (the case of interest when doing one-loop calculations) numerous approaches have been employed to determine this expansion to very high order; when $x \neq y$ (relevant for doing calculations beyond one-loop) there appear to be but two examples of performing the DeWitt expansion. In this paper we compute the off-diagonal elements of the DeWitt expansion coefficients using the Fock-Schwinger gauge. Our technique is based on representing $M_{x y}$ by a quantum mechanical path integral. We also generalize our method to the case of curved space, allowing us to determine the DeWitt expansion of $\tilde{M}_{x y}=\left\langle x\left|\exp \frac{1}{2}\left[\frac{1}{\sqrt{g}}\left(\partial_{\mu}-i A_{\mu}\right) g^{\mu \nu} \sqrt{g}\left(\partial_{\nu}-i A_{\nu}\right)\right] t\right| y\right\rangle$ by use of normal coordinates. By comparison with results for the DeWitt expansion of this matrix element obtained by the iterative solution of the diffusion equation, the relative merit of different approaches to the representation of $\tilde{M}_{x y}$ as a quantum mechanical path integral can be assessed. Furthermore, the exact dependence of $\tilde{M}_{x y}$ on some geometric scalars can be determined. In two appendices, we discuss boundary effects in the one-dimensional quantum mechanical path integral, and the curved space generalization of the Fock-Schwinger gauge.
\end{abstract}

04.62+v, 11.10.Jj, 11.15.Bt

Typeset using REVTEX

*email: tmleafs@apmaths.uwo.ca 


\section{INTRODUCTION}

There is a long history of computing the elements $a_{n}\left(x_{0}, \Delta\right)$ in the expansion of $M_{x y}$ in powers of $t$,

$$
\begin{gathered}
M_{x y} \equiv\left\langle x\left|\exp \left\{-\left[\frac{1}{2}(p-A)^{2}+V\right] t\right\}\right| y\right\rangle \\
=\frac{e^{-\Delta^{2} / 2 t}}{(2 \pi t)^{D / 2}} \sum_{n=0}^{\infty} a_{n}\left(x_{0}, \Delta\right) t^{n} \\
\left(D=\text { no. of dimensions, } p=-i \partial, x_{0}=(x+y) / 2, \Delta=x-y\right),
\end{gathered}
$$

in the limit $\Delta=0$ [1]. This expansion when $\Delta=0$ is extremely useful when examining certain properties of the generating functional at one-loop order; in particular, the divergence structure of a theory at one-loop order can be discerned. Among the approaches used to evaluate $a_{n}\left(x_{0}, 0\right)$ are the perturbative solution to the heat equation [1],2], the use of pseudo-differential operators [3], working in momentum space [4], systematically rearranging a Schwinger expansion of (1a) in powers of $A$ and $V$ into an expression of the form (1b) [5.6] and representing (1a) as a quantum mechanical path integral (QMPI) hence expanding it in powers of $t\left[7,[8]\right.$. The only places of which the authors are aware where $a_{n}\left(x_{0}, \Delta\right)$ is considered for $\Delta \neq 0$ are in [2] and [6]. These coefficients are useful in considering multi-loop processes [9], which motivates us to pursue them further. The quantum mechanical path integral has proved useful in computing Green's functions at one-loop order [10 13] and beyond [14 [16]; this suggests using this approach to examine $a_{n}\left(x_{0}, \Delta\right)$ for $\Delta \neq 0$. Although our method is not identical to that of [f], the two approaches are similar and both results agree when $\Delta=0$.

The representation of

$$
\tilde{M}_{x y}=\left\langle x\left|\exp \left\{\frac{1}{2}\left[\frac{1}{\sqrt{g}}\left(\partial_{\mu}-i A_{\mu}\right) g^{\mu \nu} \sqrt{g}\left(\partial_{\nu}-i A_{\nu}\right)\right] t\right\}\right| y\right\rangle
$$

in terms of a quantum mechanical path integral is not uniquely specified [17 [9], as discussed in [14]. We use one of the various forms of the QMPI to expand $\tilde{M}_{x y}$ and compare our results with those of [2]. Furthermore, a partial summation of the DeWitt expansion to obtain the full dependence of $\tilde{M}_{x y}$ on $R$ and $R_{\alpha \beta} \Delta^{\alpha} \Delta^{\beta}$ is possible [2].

\section{EXPANDING $M_{X Y}$}

It is possible to represent $M_{x y}$ as an appropriately normalized QMPI [21],

$$
M_{x y}=\int_{y}^{x} D q(\tau) \mathcal{P} \exp \int_{0}^{t} d \tau\left\{-\frac{\dot{q}^{2}(\tau)}{2}+i \dot{q}(\tau) \cdot A(q(\tau))-V(q(\tau))\right\}
$$

where path-ordered integration is implied over trajectories with end points $q(0)=y$ and $q(t)=x$.

We attempt to construct a power series about some point $x_{0}$ which we arbitrarily choose to be the mid-point between $x$ and $y$. Defining the relative coordinate $\delta$ by 


$$
q(\tau)=x_{0}+\delta(\tau),
$$

and imposing the Fock-Schwinger gauge condition [22],

$$
\delta(\tau) \cdot A\left(x_{0}+\delta(\tau)\right)=0,
$$

one can expand the gauge field in powers of $\delta$,

$$
\begin{aligned}
A_{\mu}\left(x_{0}+\delta(\tau)\right) & =\int_{0}^{1} d \alpha \alpha \delta^{\lambda}(\tau) F_{\lambda \mu}\left(x_{0}+\alpha \delta(\tau)\right) \\
& =\sum_{N=0}^{\infty} \frac{1}{N !(N+2)}\left[\delta(\tau) \cdot D\left(x_{0}\right)\right]^{N} \delta^{\lambda}(\tau) F_{\lambda \mu}\left(x_{0}\right) .
\end{aligned}
$$

The scalar potential can be similarly expanded,

$$
V\left(x_{0}+\delta(\tau)\right)=\sum_{N=0}^{\infty} \frac{1}{N !}\left[\delta(\tau) \cdot D\left(x_{0}\right)\right]^{N} V\left(x_{0}\right) .
$$

Here gauge-covariant differentiation at $x_{0}$ has been denoted by $D\left(x_{0}\right)$. Together, (5b) and (6) allow (10) to be written as

$$
\begin{aligned}
M_{x y}= & \int_{-\Delta / 2}^{\Delta / 2} D \delta(\tau) \exp \left[-\int_{0}^{t} d \tau \frac{\dot{\delta}^{2}(\tau)}{2}\right] \sum_{L=0}^{\infty} \frac{1}{L !} \\
& \times \mathcal{P}\left\{\sum_{N=0}^{\infty} \frac{1}{N !} \int_{0}^{t} d \tau\left[\delta(\tau) \cdot D\left(x_{0}\right)\right]^{N}\left[\frac{i}{N+2} \dot{\delta}^{\mu}(\tau) \delta^{\lambda}(\tau) F_{\lambda \mu}\left(x_{0}\right)-V\left(x_{0}\right)\right]\right\}^{L} .
\end{aligned}
$$

In the above expansion we intend to treat all terms in the expansions of the potentials as perturbations on the free-field action $\frac{1}{2} \int_{0}^{t} d \tau \dot{\delta}^{2}(\tau)$. By contrast, the authors of reference [23] have shown how, through the introduction of an appropriate tensor basis, one can include the lowest-order term of the derivative expansion of the electromagnetic field in a nonperturbative fashion. Although the latter technique could be applied here, we adopt the purely perturbative approach which is algebraically simpler and more suitable for the purpose of illustration; furthermore, we are free to consider a non-abelian gauge group.

The path integral in (7) can be evaluated by systematic functional differentiation of the standard result [21,24, 12]

$$
\begin{aligned}
& \int_{-\Delta / 2}^{\Delta / 2} D \delta(\tau) \exp \int_{0}^{t} d \tau\left\{-\frac{\dot{\delta}^{2}(\tau)}{2}+\gamma(\tau) \cdot \delta(\tau)\right\} \\
& =\frac{e^{-\Delta^{2} / 2 t}}{(2 \pi t)^{D / 2}} \exp \left\{\int_{0}^{t} d \tau\left(-\frac{1}{2}+\frac{\tau}{t}\right) \Delta \cdot \gamma(\tau)-\frac{1}{2} \int_{0}^{t} d \tau d \tau^{\prime} G\left(\tau, \tau^{\prime}\right) \gamma(\tau) \cdot \gamma\left(\tau^{\prime}\right)\right\},
\end{aligned}
$$

with respect to $\gamma_{\alpha}(\tau)$ and then setting $\gamma=0$. (Here, $G\left(\tau, \tau^{\prime}\right) \equiv \frac{1}{2}\left|\tau-\tau^{\prime}\right|-\frac{1}{2}\left(\tau+\tau^{\prime}\right)+\frac{\tau \tau^{\prime}}{t}$ is the Green's function of a free particle on the worldline.(1) For example, after two such derivatives, it is easily shown that

\footnotetext{
${ }^{1}$ In appendix A we address some concerns about the validity of this Green's function on the finite interval $[0, t]$. The complications presented there are not expected to contribute in this flat-space limit.
} 


$$
\begin{aligned}
& \int_{-\Delta / 2}^{\Delta / 2} D \delta(\tau) \delta^{\alpha}\left(\tau_{a}\right) \delta^{\beta}\left(\tau_{b}\right) \exp \left[-\int_{0}^{t} d \tau \frac{\dot{\delta}^{2}(\tau)}{2}\right] \\
& =\frac{e^{-g_{\mu \nu} \Delta^{\mu} \Delta^{\nu} / 2 t}}{(2 \pi t)^{D / 2}}\left[-G\left(\tau_{a}, \tau_{b}\right) g^{\alpha \beta}+\left(-\frac{1}{2}+\frac{\tau_{a}}{t}\right) \Delta^{\alpha}\left(-\frac{1}{2}+\frac{\tau_{b}}{t}\right) \Delta^{\beta}\right] .
\end{aligned}
$$

From (8), it is easily seen that no term in (7) will involve factors of $\Delta^{2}$. From this observation, combined with simple power counting arguments, it is straightforward to tabulate the possible contributions to the various coefficients $a_{n}\left(x_{0}, \Delta\right)$ (see table $\mathbb{\mathbb { Q }}$ ). (When a temperature dependent QMPI is considered as in [12], then the temperature provides a second dimensionful parameter which must be considered). The coefficients of the various contributions can be easily determined by appropriately choosing $L$ and $N$ in (7) and then systematically applying (8). For example, if $L=n, N=0$, then it is apparent that the contribution to $a_{n}\left(x_{0}, \Delta\right)$ proportional to $V^{n}\left(x_{0}\right)$ is $\frac{1}{n !}\left(-V\left(x_{0}\right)\right)^{n}$. By setting $L=N=1$ in (7), we find after a very short calculation that the contribution to $a_{1}\left(x_{0}, \Delta\right)$ proportional to $\Delta^{\alpha} D_{\beta} F_{\alpha}{ }^{\beta}$ is $\frac{-i}{12} \Delta^{\alpha} D_{\beta} F_{\alpha}{ }^{\beta}$. With $L=2, N=0$, the contribution to $M_{x y}$ is straightforwardly computed to be

$$
M_{x y}^{(2,0)}=\frac{e^{-\Delta^{2} / 2 t}}{(2 \pi t)^{D / 2}}\left(-\frac{t}{24} \Delta^{\alpha} \Delta^{\beta} F_{\alpha \mu} F_{\beta}{ }^{\mu}-\frac{t^{2}}{48} F_{\alpha \beta} F^{\alpha \beta}\right),
$$

giving a contribution to both $a_{1}\left(x_{0}, \Delta\right)$ and $a_{2}\left(x_{0}, \Delta\right)$. These results are all consistent with the flat space limit of the expressions for $a_{n}\left(x_{0}, \Delta\right)$ given in [2].

\section{EXPANDING $\tilde{M}_{X Y}$}

As has been noted in the introduction and in [14], there are various representations of the matrix element

$$
\tilde{M}_{x y}=\left\langle x\left|\exp \left\{\frac{1}{2}\left[\frac{1}{\sqrt{g}}\left(\partial_{\mu}-i A_{\mu}\right) g^{\mu \nu} \sqrt{g}\left(\partial_{\nu}-i A_{\nu}\right)\right] t\right\}\right| y\right\rangle
$$

in terms of a QMPI. We adopt the approach of [19] in which the QMPI is computed using a normal coordinate expansion of the coordinate being integrated over and the form of the classical action is fixed by having $\tilde{M}_{x y}$ satisfy the appropriate heat kernel equation. (This representation does not coincide with the expression given in [25] for any value of the parameter $p$ appearing there.) This representation gives a dependence of $\tilde{M}_{x y}$ on $R$ that coincides with that of [20,2], and agrees, to all orders so far checked, with the results of [2]. The full dependence of $M_{x y}$ on $R_{\alpha \beta} \Delta^{\alpha} \Delta^{\beta}$ can also be determined.

We are now faced with evaluating

$$
\begin{gathered}
\tilde{M}_{x y}=\int_{y}^{x} D q^{\alpha}(\tau) \sqrt{g(q(\tau))} \mathcal{P} \exp \left\{-\int_{0}^{t} d \tau\left[\frac{1}{2} g_{\mu \nu}(q(\tau)) \dot{q}^{\mu}(\tau) \dot{q}^{\nu}(\tau)\right.\right. \\
\left.\left.-i \dot{q}^{\mu}(\tau) A_{\mu}(q(\tau))+\frac{1}{8} R(q(\tau))\right]\right\} .
\end{gathered}
$$

The factor $\sqrt{g(q(\tau))}=\operatorname{det}^{1 / 2} g_{\mu \nu}(q(\tau))$ in the measure also occurs in the non-linear sigma model [26], but there it is usually discarded as it gives a contribution to the effective action 
that is proportional to $\delta(0)$ which, when regulated using dimensional regularization [27] or operator regularization [28], goes to zero. We are dealing with a model in which no regularization is required; indeed it turns out that divergent contributions from $\sqrt{g}$ are essential to render the path integral in (11) well-defined. It is most convenient to incorporate the effects of $\sqrt{g}$ by using ghosts as was done in [19]. Perhaps the simplest way to do this is to introduce a vector of real Bosonic fields $b_{\alpha}(\tau)$ which vanish at the end points $(\tau=0, t)$. (The structure of the ghost sector is not unique, and the authors of [19] opt, instead, to use either a pair of Fermionic scalars or a contravariant Bosonic ghost $b^{\alpha}$ and two contravariant Fermionic ghosts $c^{\alpha}$ and $\bar{c}^{\alpha}$.) Then, equation (11) can be re-expressed as

$$
\begin{aligned}
\tilde{M}_{x y}=\int_{y}^{x} D q^{\alpha}(\tau) & \int_{0}^{0} D b_{\beta}(\tau) \mathcal{P} \exp \left\{-\int_{0}^{t} d \tau\left[\frac{1}{2} g^{\mu \nu} b_{\mu}(\tau) b_{\nu}(\tau)\right.\right. \\
& \left.\left.+\frac{1}{2} g_{\mu \nu}(q(\tau)) \dot{q}^{\mu}(\tau) \dot{q}^{\nu}(\tau)-i \dot{q}^{\alpha}(\tau) A_{\alpha}(q(\tau))+\frac{1}{8} R(q(\tau))\right]\right\} .
\end{aligned}
$$

A normal coordinate expansion [29] is now made about a point $\phi(\tau)$ so that

$$
q^{\alpha}(\tau)=\phi^{\alpha}(\tau)+\pi^{\alpha}(\xi(\tau))
$$

Following the developments of [27], we have

$$
\begin{aligned}
& R(q(\tau))=R(\phi(\tau))+\frac{1}{1 !} R_{; \alpha}(\phi(\tau)) \xi^{\alpha}(\tau)+\frac{1}{2 !} R_{; \alpha \beta}(\phi(\tau)) \xi^{\alpha}(\tau) \xi^{\beta}(\tau)+\cdots \\
& g_{\mu \nu}(q(\tau))= g_{\mu \nu}(\phi(\tau))+\frac{1}{3} R_{\mu \alpha \beta \nu}(\phi(\tau)) \xi^{\alpha}(\tau) \xi^{\beta}(\tau)+\frac{1}{6} R_{\mu \alpha \beta \nu ; \gamma}(\phi(\tau)) \xi^{\alpha}(\tau) \xi^{\beta}(\tau) \xi^{\gamma}(\tau) \\
&+\left(\frac{1}{20} R_{\mu \alpha \beta \nu ; \gamma \delta}(\phi(\tau))+\frac{2}{45} R_{\mu \alpha \beta \sigma}(\phi(\tau)) R_{\gamma \delta \nu}^{\sigma}(\phi(\tau))\right) \xi^{\alpha}(\tau) \xi^{\beta}(\tau) \xi^{\gamma}(\tau) \xi^{\delta}(\tau) \\
&+\cdots \\
& g^{\mu \nu}(q(\tau))= g^{\mu \nu}(\phi(\tau))-\frac{1}{3} R_{\alpha \beta}^{\mu}(\phi(\tau)) \xi^{\alpha}(\tau) \xi^{\beta}(\tau)-\frac{1}{6} R_{\alpha \beta}^{\mu}{ }_{\alpha \gamma}{ }^{\nu}(\phi(\tau)) \xi^{\alpha}(\tau) \xi^{\beta}(\tau) \xi^{\gamma}(\tau) \\
&+\left(-\frac{1}{20} R_{\alpha \beta}^{\mu}{ }^{\nu}{ }_{; \gamma \delta}(\phi(\tau))+\frac{3}{45} R^{\mu}{ }_{\alpha \beta \sigma}(\phi(\tau)) R_{\gamma \delta}^{\sigma}{ }^{\nu}(\phi(\tau))\right) \xi^{\alpha}(\tau) \xi^{\beta}(\tau) \xi^{\gamma}(\tau) \xi^{\delta}(\tau) \\
&+\cdots,
\end{aligned}
$$

and

$$
\begin{aligned}
\dot{q}^{\mu}(\tau)= & \dot{\phi}^{\mu}(\tau)+D_{\tau} \xi^{\mu}(\tau)+\frac{1}{3} R^{\mu}{ }_{\alpha \beta \gamma}(\phi(\tau)) \xi^{\alpha}(\tau) \xi^{\beta}(\tau) \dot{\phi}^{\gamma}(\tau)+\cdots \\
& \left(D_{\tau} \xi^{\mu}(\tau) \equiv \dot{\xi}^{\mu}(\tau)+\Gamma_{\beta \gamma}^{\mu} \xi^{\beta}(\tau) \dot{\phi}^{\gamma}(\tau)\right)
\end{aligned}
$$

As shown in appendix $B$, by imposing a gauge condition analogous to (舟), one finds that the corresponding normal coordinate expansion for the gauge field is (B3)

$$
\begin{aligned}
A_{\mu}(q(\tau))= & \frac{1}{2} F_{\alpha \mu}(\phi(\tau)) \xi^{\alpha}(\tau)+\frac{1}{3} F_{\alpha \mu ; \beta}(\phi(\tau)) \xi^{\alpha}(\tau) \xi^{\beta}(\tau) \\
& +\left(\frac{1}{8} F_{\alpha \mu ; \beta \gamma}(\phi(\tau))+\frac{1}{24} F_{\alpha \sigma}(\phi(\tau)) R_{\beta \gamma \mu}^{\sigma}(\phi(\tau))\right) \xi^{\alpha}(\tau) \xi^{\beta}(\tau) \xi^{\gamma}(\tau) \\
& +\left(\frac{1}{30} F_{\alpha \mu ; \beta \gamma \delta}(\phi(\tau))+\frac{1}{60} F_{\alpha \sigma}(\phi(\tau)) R_{\beta \gamma \mu ; \delta}^{\sigma}(\phi(\tau))\right. \\
& \left.\quad+\frac{1}{30} F_{\alpha \sigma ; \delta}(\phi(\tau)) R_{\beta \gamma \mu}^{\sigma}(\phi(\tau))\right) \xi^{\alpha}(\tau) \xi^{\beta}(\tau) \xi^{\gamma}(\tau) \xi^{\delta}(\tau)+\cdots
\end{aligned}
$$


If we take $\phi(\tau)$ to be the geodesic mid-point, $x_{0}$, between $x$ and $y$, then $\dot{\phi}^{\alpha}(\tau)$ vanishes and the above expansions simplify a bit. Letting $\Delta$ denote the difference between the normal coordinates of $x$ and $y$, equation (12) becomes

$$
\begin{aligned}
\tilde{M}_{x y}=\int_{-\Delta / 2}^{\Delta / 2} D \xi^{\alpha}(\tau) \int_{0}^{0} D & b_{\beta}(\tau) \exp \left\{-\int_{0}^{t} d \tau\left[\frac{1}{2} g_{\mu \nu} \dot{\xi}^{\mu}(\tau) \dot{\xi}^{\nu}(\tau)+\frac{1}{2} g^{\mu \nu} b_{\mu}(\tau) b_{\nu}(\tau)\right]\right\} e^{-R t / 8} \\
\times \mathcal{P} \sum_{N=0}^{\infty} \frac{1}{N !}\left\{-\int_{0}^{t}\right. & d \tau\left[\frac{1}{2}\left(\frac{1}{3} R_{\mu \alpha \beta \nu} \xi^{\alpha}(\tau) \xi^{\beta}(\tau)+\frac{1}{6} R_{\mu \alpha \beta \nu ; \gamma} \xi^{\alpha}(\tau) \xi^{\beta}(\tau) \xi^{\gamma}(\tau)+\cdots\right) \dot{\xi}^{\mu}(\tau) \dot{\xi}^{\nu}(\tau)\right. \\
& +\frac{1}{2}\left(-\frac{1}{3} R^{\mu}{ }_{\alpha \beta}{ }^{\nu} \xi^{\alpha}(\tau) \xi^{\beta}(\tau)-\frac{1}{6} R^{\mu}{ }_{\alpha \beta}{ }^{\nu}{ }_{; \gamma} \xi^{\alpha}(\tau) \xi^{\beta}(\tau) \xi^{\gamma}(\tau)+\cdots\right) b_{\mu}(\tau) b_{\nu}(\tau) \\
& -i\left(\frac{1}{2} F_{\alpha \mu} \xi^{\alpha}(\tau)+\frac{1}{3} F_{\alpha \mu ; \beta} \xi^{\alpha}(\tau) \xi^{\beta}(\tau)+\cdots\right) \dot{\xi}^{\mu} \\
& \left.\left.+\frac{1}{8}\left(R_{; \alpha} \xi^{\alpha}(\tau)+\frac{1}{2} R_{; \alpha \beta} \xi^{\alpha}(\tau) \xi^{\beta}(\tau)+\cdots\right)\right]\right\}^{N} .
\end{aligned}
$$

(All geometrical and gauge quantities in (15) are evaluated at $x_{0}$.)

The standard results

$$
\begin{aligned}
& \int_{-\Delta / 2}^{\Delta / 2} D \xi^{\alpha}(\tau) \exp \left\{\int_{0}^{t} d \tau\left(-\frac{1}{2} g_{\mu \nu} \dot{\xi}^{\mu}(\tau) \dot{\xi}^{\nu}(\tau)+\xi^{\mu}(\tau) \gamma_{\mu}(\tau)\right)\right\} \\
= & \frac{e^{-g_{\mu \nu} \Delta^{\mu} \Delta^{\nu} / 2 t}}{(2 \pi t)^{D / 2} \sqrt{g}} \exp \left\{\int_{0}^{t} d \tau\left(-\frac{1}{2}+\frac{\tau}{t}\right) \Delta^{\mu} \gamma_{\mu}(\tau)-\frac{1}{2} \int_{0}^{\tau} d \tau d \tau^{\prime} G\left(\tau, \tau^{\prime}\right) g^{\mu \nu} \gamma_{\mu}(\tau) \gamma_{\nu}\left(\tau^{\prime}\right)\right\}
\end{aligned}
$$

and,

$$
\begin{aligned}
& \int_{0}^{0} D b_{\alpha}(\tau) \exp \left\{\int_{0}^{t} d \tau\left(-\frac{1}{2} g^{\mu \nu} b_{\mu}(\tau) b_{\nu}(\tau)+b_{\mu}(\tau) B^{\mu}(\tau)\right)\right\} \\
& =\sqrt{g} \exp \left\{\frac{1}{2} \int_{0}^{t} d \tau d \tau^{\prime} G^{\text {ghost }}\left(\tau, \tau^{\prime}\right) g_{\mu \nu} B^{\mu}(\tau) B^{\nu}\left(\tau^{\prime}\right)\right\}_{B(0)=B(t)=0}
\end{aligned}
$$

permit one to compute the functional integrals appearing in (15). Equivalently, one can generate the necessary contractions using the formalism of equation (A2). In either case, one should be careful to use the Green's functions of equations (A6) (or in simple cases, equations (A8)) as discussed in appendix A.

For example, if we restrict our attention to the contribution to (15) that is linear in $R_{\mu \alpha \nu \beta}$, we have

$$
\begin{array}{rl}
\tilde{M}_{x y}^{R}=\int_{-\Delta / 2}^{\Delta / 2} & D \xi^{\alpha}(\tau) \int_{0}^{0} D b_{\beta}(\tau) e^{-R t / 8} \\
& \times \exp \left\{-\int_{0}^{t} d \tau\left[\frac{1}{2} g_{\mu \nu} \dot{\xi}^{\mu}(\tau) \dot{\xi}^{\nu}(\tau)+\frac{1}{2} g^{\mu \nu} b_{\mu}(\tau) b_{\nu}(\tau)\right]\right\} \\
& \times\left\{-\frac{1}{6} R_{\mu \alpha \beta \nu} \int_{0}^{t} d \tau \xi^{\alpha}(\tau) \xi^{\beta}(\tau) \dot{\xi}^{\mu}(\tau) \dot{\xi}^{\nu}(\tau)+\frac{1}{6} R^{\mu}{ }_{\alpha \beta}^{\nu} \int_{0}^{t} d \tau \xi^{\alpha}(\tau) \xi^{\beta}(\tau) b_{\mu}(\tau) b_{\nu}(\tau)\right\} \\
= & \frac{e^{-g_{\mu \nu} \Delta^{\mu} \Delta^{\nu} / 2 t}}{(2 \pi t)^{D / 2}} e^{-R t / 8} \int_{0}^{t} d \tau\left\{-\frac{1}{2}\left[g^{\mu \nu} g^{\alpha \beta} G(\dot{\tau}, \dot{\tau}) G(\tau, \tau)+\left(g^{\mu \alpha} g^{\nu \beta}+g^{\mu \beta} g^{\nu \alpha}\right) G^{2}(\tau, \dot{\tau})\right.\right. \\
& -\Delta^{\mu} \Delta^{\nu}\left(\frac{1}{t}\right)^{2} g^{\alpha \beta} G(\tau, \tau)-\Delta^{\alpha} \Delta^{\beta} g^{\mu \nu}\left(-\frac{1}{2}+\frac{\tau}{t}\right)^{2} G(\dot{\tau}, \dot{\tau})
\end{array}
$$




$$
\begin{aligned}
& -\left(\Delta^{\mu} \Delta^{\alpha} g^{\nu \beta}+\Delta^{\nu} \Delta^{\beta} g^{\mu \alpha}+\Delta^{\mu} \Delta^{\beta} g^{\nu \alpha}+\Delta^{\nu} \Delta^{\alpha} g^{\mu \beta}\right) \frac{1}{t}\left(-\frac{1}{2}+\frac{\tau}{t}\right) G(\tau, \dot{\tau}) \\
& \left.+\Delta^{\alpha} \Delta^{\beta} \Delta^{\mu} \Delta^{\nu}\left(-\frac{1}{2}+\frac{\tau}{t}\right)^{2}\left(\frac{1}{t}\right)^{2}\right] \\
+ & {\left.\left[-G(\tau, \tau) g^{\alpha \beta}+\left(-\frac{1}{2}+\frac{\tau}{t}\right)^{2} \Delta^{\alpha} \Delta^{\beta}\right] G^{\text {ghost }}(\tau, \tau) g^{\mu \nu}\right\}\left(\frac{1}{6} R_{\mu \alpha \beta \nu}\right) . }
\end{aligned}
$$

Here, a dot over an argument of the Green's function $G$ indicates differentiation with respect to that argument. As explained in appendix A, these derivatives cannot always be obtained at the end points $(\tau=0, t)$ by naive differentiation of $G\left(\tau, \tau^{\prime}\right)$ (c.f. equation (A8d)). When the explicit form of $G\left(\tau, \tau^{\prime}\right)$ and its derivatives are substituted into (17), we are left with with

$$
\begin{aligned}
\tilde{M}_{x y}^{R}= & \frac{e^{-g_{\mu \nu} \Delta^{\mu} \Delta^{\nu} / 2 t}}{(2 \pi t)^{D / 2}} \frac{1}{6} e^{-R t / 8} \int_{0}^{t} d \tau \\
& \quad \times\left\{R_{\alpha \beta} \Delta^{\alpha} \Delta^{\beta}\left[\frac{1}{t^{2}}\left(-\tau+\frac{\tau^{2}}{t}\right)-\frac{1}{t}\left(-\frac{1}{2}+\frac{\tau}{t}\right)^{2}-[\delta(2 \tau)+\delta(2 t-2 \tau)]\left(-\frac{1}{2}+\frac{\tau}{t}\right)^{2}\right]\right. \\
& \left.\quad+R\left[-\frac{1}{t}\left(-\tau+\frac{\tau^{2}}{t}\right)+\left(-\frac{1}{2}+\frac{\tau}{t}\right)^{2}+[\delta(2 \tau)+\delta(2 t-2 \tau)]\left(-\tau+\frac{\tau^{2}}{t}\right)\right]\right\} \\
= & \frac{e^{-g_{\mu \nu} \Delta^{\mu} \Delta^{\nu} / 2 t}}{(2 \pi t)^{D / 2}} e^{-R t / 8}\left[-\frac{1}{12} R_{\alpha \beta} \Delta^{\alpha} \Delta^{\beta}+\frac{1}{24} R t\right]
\end{aligned}
$$

where $R_{\alpha \beta}=R_{\alpha \beta \mu}^{\mu}$ and $R=R_{\alpha \beta} g^{\alpha \beta}$. (Notice that all dependence on $\delta(0)$ in (17) has cancelled out due to the compensating contributions from the ghost-fields.) It is easily seen that the entire dependence of $\tilde{M}_{x y}$ on $R$ and $R_{\alpha \beta} \Delta^{\alpha} \Delta^{\beta}$ is given by

$$
\begin{aligned}
\tilde{M}_{x y}^{(R, \Delta \cdot R \cdot \Delta)} & =\frac{e^{-g_{\mu \nu} \Delta^{\mu} \Delta^{\nu} / 2 t}}{(2 \pi t)^{D / 2}} \sum_{N=0}^{\infty} \frac{e^{-R t / 8}}{N !}\left[\frac{-R_{\alpha \beta} \Delta^{\alpha} \Delta^{\beta}}{12}+\frac{R t}{24}\right]^{N} \\
& =\frac{e^{-g_{\mu \nu} \Delta^{\mu} \Delta^{\nu} / 2 t}}{(2 \pi t)^{D / 2}} \exp \left[-\frac{R t}{12}-\frac{R_{\alpha \beta} \Delta^{\alpha} \Delta^{\beta}}{12}\right] .
\end{aligned}
$$

The dependence of $\tilde{M}_{x y}$ on both $R$ and $R_{\alpha \beta} \Delta^{\alpha} \Delta^{\beta}$ in (19) agrees with that of 20,2,19] (once the different normalization of $t$ is taken into account).

A completely analogous calculation can be used to fix the lowest order dependence on $R_{\alpha \beta ; \gamma} \Delta^{\alpha} \Delta^{\beta} \Delta^{\gamma}$; it is found to vanish. This also appears to be consistent with the results of [2] (where the coefficient $R_{\alpha \beta ; \gamma}$ is evaluated at the end-point $y$, instead of the mid-point $x_{0}$ ). Further terms in the DeWitt expansion of $\tilde{M}_{x y}$ can be similarly determined.

The techniques used in this section may be employed to find the effective action for a particle moving in a gravitational field [30]. This involves taking $\phi^{\alpha}(\tau)$ to be arbitrary in (13) rather than restricting it to be $x_{0}$.

\section{DISCUSSION}

In the preceding sections we have considered how the QMPI can be used to determine the elements of the DeWitt expansion for the heat kernel both on and off the diagonal. This

technique is seen to be easier to use than the original approach [6] in which the heat equation 
was solved perturbatively. By employing the off-diagonal elements, calculations can be done to two-loop order [9]. The method works in both flat and curved space.

Because of the simplicity of the proper-time $(\tau)$ integrands which arise when implementing this method, we expect that high-order covariant expansions could be computerized using presently available symbolic algebra packages. This approach has already been employed in the diagonal case [7]. In the off-diagonal case, surface terms (proportional to $\Delta$ ) introduce an additional combinatorical consideration which should be tractable.

Finally, it is worth noting, that although our treatment of this problem focused specifically on the case where the vector potential $A_{\mu}$ was a gauge field, this is unnecessarily restrictive and the methods presented here would work equally well for a general vector coupling to a background field. For example, if one were interested in radiative corrections to Fermionic Green's functions in flat-space QED, the following quantum mechanical operator would be of interest [31]

$$
\left(\begin{array}{ccc}
p^{2} g_{\mu \nu}-\left(1-\frac{1}{a}\right) p_{\mu} p_{\nu} & -\left(\gamma_{\mu} \psi\right)^{T} & \left(\bar{\psi} \gamma_{\mu}\right) \\
\left(\gamma_{\nu} \psi\right) & 0 & \not p-m \\
-\left(\bar{\psi} \gamma_{\nu}\right)^{T} & -(\not p-m)^{T} & 0
\end{array}\right)_{(\mu, \nu)}
$$

By factoring in the constant supermatrix

$$
\left(\begin{array}{ccc}
g_{\nu \lambda} & 0 & 0 \\
0 & 0 & (\not p+m)^{T} \\
0 & -(\not p+m) & 0
\end{array}\right)_{(\nu, \lambda)}
$$

and choosing the $a=1$ gauge, operator (20) becomes

$$
\left(\begin{array}{ccc}
p^{2} g_{\mu \lambda} & -\left(\bar{\psi} \gamma_{\mu}\right)(\not p+m) & -\left(\gamma_{\mu} \psi\right)^{T}(\not p+m)^{T} \\
\left(\gamma_{\lambda} \psi\right) & p^{2}+m^{2} & 0 \\
-\left(\bar{\psi} \gamma_{\lambda}\right)^{T} & 0 & p^{2}+m^{2}
\end{array}\right)_{(\mu, \lambda)}
$$

After completing the square of $p$ (and noting that $p^{T}=-p$ in the coordinate-space representation), the heat kernel of this operator is easily shown to have the form of equation (1a) with vector potential,

$$
A_{\nu}(q)=\frac{1}{2}\left(\begin{array}{ccc}
0 & \bar{\psi}(q) \gamma_{\mu} \gamma_{\nu} & -\left(\gamma_{\nu} \gamma_{\mu} \psi(q)\right)^{T} \\
0 & 0 & 0 \\
0 & 0 & 0
\end{array}\right)_{(\mu, \lambda)}
$$

and scalar potential,

$$
V(q)=\left(\begin{array}{ccc}
0 & \left(\bar{\psi}(q) \gamma_{\mu}\right)\left(\frac{i}{2} \overleftarrow{\not}-m\right) & {\left[\left(\frac{-i}{2} \not \partial-m\right) \gamma_{\mu} \psi(q)\right]^{T}} \\
\left(\gamma_{\lambda} \psi(q)\right) & m^{2} & 0 \\
-\left(\bar{\psi}(q) \gamma_{\lambda}\right)^{T} & 0 & m^{2}
\end{array}\right)_{(\mu, \lambda)}
$$

After the appropriate Taylor expansions of these potentials is substituted into eq. (2) the method should proceed in the obvious way. 


\section{ACKNOWLEDGMENTS}

We would like to thank the Natural Science and Engineering Research Council of Canada (NSERC) for financial support.

D.G.C.M. would also like to thank the Dublin Institute for Advanced Study for its hospitality while much of this work was being done. He would also like to thank $\mathrm{C}$. Wiesendanger for having pointed out ref. [2].

F.A.D. would like to thank D.J. O'Connor for providing materials related to appendix $\mathbb{B}$ and M. Lüscher for a communication regarding the results of ref. [2].

\section{APPENDIX A: EVALUATION OF THE PATH INTEGRAL}

Here we explicitly evaluate the path integrals used in the previous sections. Firstly, we define the type of integrals which need to be evaluated to obtain the expectation value of a function $F$ of dynamical variables $\xi_{\mu}(\tau)$ and $b^{\mu}(\tau)$,

$$
\begin{aligned}
\langle F(\xi, b)\rangle_{\Delta} \equiv & \left\langle\frac{\Delta}{2}, 0|F(\xi, b)|-\frac{\Delta}{2}, 0\right\rangle \\
= & {\left[\frac{e^{-\Delta^{\mu} \Delta^{\nu} g_{\mu \nu} / 2 t}}{(2 \pi t)^{D / 2}}\right]^{-1} \int_{-\Delta / 2}^{\Delta / 2} D \xi^{\alpha}(\tau) \int_{0}^{0} D b_{\beta}(\tau) F(\xi, b) } \\
& \times \exp \left\{-\int_{0}^{t} d \tau\left[\frac{1}{2} g_{\mu \nu} \dot{\xi}^{\mu}(\tau) \dot{\xi}^{\nu}(\tau)+\frac{1}{2} g^{\mu \nu} b_{\mu}(\tau) b_{\nu}(\tau)\right]\right\},
\end{aligned}
$$

which satisfies the normalization condition $\langle 1\rangle_{\Delta}=1$.

We eliminate the boundary parameter $\Delta$ from the path integral by integrating over fluctuations about the classical geodesic $\xi_{\mathrm{cl}}(\tau)=(-1 / 2+\tau / t) \Delta$. Letting $\xi \rightarrow \xi+\xi_{\mathrm{cl}}$ it is easily shown that

$$
\langle F[\xi, b]\rangle_{\Delta}=\left\langle F\left[\xi+\xi_{\mathrm{cl}}, b\right]\right\rangle_{0}
$$

Thus, for the purpose of evaluating any particular term in equation (15), it will be sufficient for us to concentrate on evaluating $\langle F[\xi, b]\rangle_{0}$ where $F$ is a monomial in $\xi, \dot{\xi}$ and $b$. We can follow the procedure usually used in applying the path integral in field theory and so we only need to evaluate the various two-point functions, $\left\langle b_{\mu}(\tau) b_{\nu}\left(\tau^{\prime}\right)\right\rangle,\left\langle\xi^{\mu}(\tau) \xi^{\nu}\left(\tau^{\prime}\right)\right\rangle$, etc, and then apply the appropriate Wick expansions.

Jumping directly into the continuum limit, one would obtain, using the standard techniques 12,24 ,

$$
\begin{aligned}
& \left\langle\xi^{\mu}(\tau) \xi^{\nu}\left(\tau^{\prime}\right)\right\rangle=-G\left(\tau, \tau^{\prime}\right) g^{\mu \nu} \\
& =-\left[\frac{1}{2}\left|\tau-\tau^{\prime}\right|-\frac{1}{2}\left(\tau+\tau^{\prime}\right)+\frac{\tau \tau^{\prime}}{t}\right] g^{\mu \nu} \\
& \begin{aligned}
\left\langle b_{\mu}(\tau) b_{\nu}\left(\tau^{\prime}\right)\right\rangle & =G^{\text {ghost }}\left(\tau, \tau^{\prime}\right) g_{\mu \nu} \\
& =\delta\left(\tau-\tau^{\prime}\right) g_{\mu \nu}
\end{aligned}
\end{aligned}
$$




$$
\left\langle\xi^{\mu}(\tau) b_{\nu}\left(\tau^{\prime}\right)\right\rangle=0
$$

Upon close inspection of the above propagators one finds that the method used for finding the explicit form of the Green's functions is problematic, especially at the end points. For example, the defining equation for $G$

$$
\frac{\partial^{2}}{\partial \tau^{2}} G\left(\tau, \tau^{\prime}\right)=\delta\left(\tau-\tau^{\prime}\right),
$$

and the corresponding boundary conditions

$$
G(\tau, 0)=G(\tau, t)=0,
$$

are contradictory at the $\tau^{\prime}=0$ and $\tau^{\prime}=t$ boundaries. Furthermore, the explicit form of the ghost propagator of equation (A3b) does not satisfy its homogeneous boundary condition at the corners $(0,0)$ and $(t, t)$.

In order to circumvent these difficulties, we adopt the approach of [19] where the path integral is taken to be the limiting case of integration over a finite set of discrete Fourier modes. The Fourier expansions

$$
\xi^{\mu}(\tau)=\sum_{n=1}^{M} \xi_{n}^{\mu} \sin \frac{n \pi \tau}{t}, \quad b_{\mu}(\tau)=\sum_{n=1}^{M} b_{\mu n} \sin \frac{n \pi \tau}{t},
$$

automatically incorporate the necessary boundary conditions in the $\Delta=0$ path integral; furthermore, they ensure that the path integral is over functions which are periodic, as required in [10]. If the cutoff $M$ is finite then the path integral over Fourier coefficients is well defined. Substituting these expansions into the action of (A1), we find that the propagators of the Fourier modes are simply [19

$$
\left\langle\xi_{n}^{\mu} \xi_{m}^{\nu}\right\rangle=\frac{2 t}{n^{2} \pi^{2}} \delta_{n m} g^{\mu \nu}, \quad\left\langle b_{\mu n} b_{\nu m}\right\rangle=\frac{2}{t} \delta_{n m} g_{\mu \nu},
$$

which we take to be our fundamental propagators. Then using (A4) the field propagators are found to be

$$
\begin{aligned}
\left\langle\xi^{\mu}(\tau) \xi^{\nu}\left(\tau^{\prime}\right)\right\rangle & =g^{\mu \nu} t \sum_{n=1}^{M} \frac{1}{n^{2} \pi^{2}}\left[\cos \frac{n \pi\left(\tau-\tau^{\prime}\right)}{t}-\cos \frac{n \pi\left(\tau+\tau^{\prime}\right)}{t}\right], \\
\left\langle b_{\mu}(\tau) b_{\nu}\left(\tau^{\prime}\right)\right\rangle & =g_{\mu \nu} \frac{1}{t} \sum_{n=1}^{M}\left[\cos \frac{n \pi\left(\tau-\tau^{\prime}\right)}{t}-\cos \frac{n \pi\left(\tau+\tau^{\prime}\right)}{t}\right], \\
\left\langle\xi^{\mu}(\tau) b_{\nu}\left(\tau^{\prime}\right)\right\rangle & =0 .
\end{aligned}
$$

These are the propagators which should be substituted into any Wick expansion of $\langle F[\xi, b]\rangle_{0}$. In principal we should attempt to approximate the continuum limit by letting $M \rightarrow \infty$ only after the proper-time integrals have been performed. Unfortunately, these harmonic expressions for the Greens functions can be unnecessarily cumbersome; in practice whenever the appropriate product of Green's functions is sufficiently well behaved as $M \rightarrow \infty$, we will sum them in that limit before substituting them into the Wick expansion. This is most 
easily done by using the variables $\tau_{ \pm}=\tau \pm \tau^{\prime}$. Then, on the relevant intervals $\tau_{+} \in[0,2 t]$, $\tau_{-} \in[-t, t]$, the following limits are easily derived from elementary Fourier theory:

$$
\begin{aligned}
\sum_{n=1}^{M} \frac{t}{n^{2} \pi^{2}} \cos \frac{n \pi \tau_{ \pm}}{t} \stackrel{M \rightarrow \infty}{\longrightarrow} \frac{1}{6} t-\frac{1}{2}\left|\tau_{ \pm}\right|+\frac{1}{4 t} \tau_{ \pm}^{2} \\
\sum_{n=1}^{M} \frac{1}{n \pi} \sin \frac{n \pi \tau_{-}}{t} \stackrel{M \rightarrow \infty}{\longrightarrow} \frac{1}{2} \operatorname{sgn} \tau_{-}-\frac{1}{2 t} \tau_{-} \\
\sum_{n=1}^{M} \frac{1}{n \pi} \sin \frac{n \pi \tau_{+}}{t} \stackrel{M \rightarrow \infty}{\longrightarrow} \frac{1}{2}-\frac{1}{2 t} \tau_{+} \\
\sum_{n=1}^{M} \frac{1}{t} \cos \frac{n \pi \tau_{-}}{t} \stackrel{M \rightarrow \infty}{\longrightarrow} \delta\left(\tau_{-}\right)-\frac{1}{2 t} \\
\sum_{n=1}^{M} \frac{1}{t} \cos \frac{n \pi \tau_{+}}{t} \stackrel{M \rightarrow \infty}{\longrightarrow} \delta\left(\tau_{+}\right)+\delta\left(2 t-\tau_{+}\right)-\frac{1}{2 t}
\end{aligned}
$$

It is thus found that in many cases the following prescription is sufficient to recover those propogators which arise in (15):

$$
\begin{aligned}
& \left\langle\xi^{\mu}(\tau) \xi^{\nu}\left(\tau^{\prime}\right)\right\rangle \rightarrow-G\left(\tau, \tau^{\prime}\right) \equiv-\left[\frac{1}{2}\left|\tau-\tau^{\prime}\right|-\frac{1}{2}\left(\tau+\tau^{\prime}\right)+\frac{\tau \tau^{\prime}}{t}\right] g^{\mu \nu} \\
& \left\langle\dot{\xi}^{\mu}(\tau) \xi^{\nu}\left(\tau^{\prime}\right)\right\rangle \rightarrow-G\left(\dot{\tau}, \tau^{\prime}\right) \equiv-\left[\frac{1}{2} \operatorname{sgn}\left(\tau-\tau^{\prime}\right)-\frac{1}{2}+\frac{\tau^{\prime}}{t}\right] g^{\mu \nu} \\
& \left\langle\dot{\xi}^{\mu}(\tau) \dot{\xi}^{\nu}\left(\tau^{\prime}\right)\right\rangle \rightarrow-G\left(\dot{\tau}, \dot{\tau}^{\prime}\right) \equiv-\left[-\delta\left(\tau-\tau^{\prime}\right)+\frac{1}{t}-\delta\left(2 t-\tau-\tau^{\prime}\right)-\delta\left(\tau+\tau^{\prime}\right)\right] g^{\mu \nu} \\
& \left\langle b_{\mu}(\tau) b_{\nu}\left(\tau^{\prime}\right)\right\rangle \rightarrow G^{\text {ghost }}\left(\tau, \tau^{\prime}\right) \equiv\left[\delta\left(\tau-\tau^{\prime}\right)-\delta\left(2 t-\tau-\tau^{\prime}\right)-\delta\left(\tau+\tau^{\prime}\right)\right] g_{\mu \nu}
\end{aligned}
$$

(We note here that (A8d) can be derived from (A8a) by requiring that $G$ be extended periodically outside of the domain $0 \leq \tau, \tau^{\prime} \leq t$, i.e $G\left(\tau+t, \tau^{\prime}+t\right) \equiv G\left(\tau, \tau^{\prime}\right)$. This periodicity is akin to the definition of the Green's function given in [10 on a compact periodic surface. A discussion of this point can be found in [15].)

Of note is the limiting case where $\tau=\tau^{\prime}$ which appears in the calculation of (17); there the following considerations apply:

- The term $\operatorname{sgn}\left(\tau-\tau^{\prime}\right)$ in (A8B) is odd in $\tau-\tau^{\prime}$ so its Fourier transform necessarily vanishes in the $\tau=\tau^{\prime}$ limit. Thus we should take $\operatorname{sgn}(0)=0$ (c.f. (A7b)).

- In the $\tau=\tau^{\prime}$ limit, the Fourier series of $\delta\left(\tau-\tau^{\prime}\right)$ in (A8d) and (A8di) leads to a regulated representation of $\delta(0)$ given by $t \delta(0)=M+1 / 2$, (c.f. (A7di)).

- Since the Fourier series of the terms $\delta(2 t-2 \tau)+\delta(2 \tau)$ (which arise in (A8d) and (A8d)) is even about the respective poles (c.f. (A7] )), any integrated contribution from these terms should be halved as a result of the poles coinciding with the end-point of the integration region, i.e.

$$
\int_{0}^{t} d \tau[\delta(2 t-2 \tau)+\delta(2 \tau)] f(\tau)=\frac{1}{4}[f(t)+f(0)] .
$$




\section{APPENDIX B: NORMAL COORDINATE EXPANSION OF THE GAUGE FIELD}

In this appendix we discuss the construction of a gauge-covariant normal coordinate expansion for the gauge potential.

By analogy with the flat-space case discussed briefly in section II and in refs. [11, [], the appropriate gauge condition for this expansion is the synchronous gauge [32] (a curvedspace generalization of the Fock-Schwinger gauge (4) [22]) which fits very well in the normal coordinate construction. In the basis of the normal coordinate system, the gauge condition is

$$
\xi^{\alpha} A_{\alpha}(\phi+\pi(\xi))=0
$$

Either by integrating along the geodesics (which is formally identical to equation (5a)) or by using differential forms [32] one can show, in the normal coordinate system, that the synchronous gauge leads to a gauge-covariant expansion for the vector potential which looks exactly like equation (5b) with the gauge-covariant normal coordinate derivative $D_{\alpha}=\frac{\partial}{\partial \xi^{\alpha}}+\left[A_{\alpha}, \ldots\right]$. The latter derivative is not covariant under reparametrization of the manifold, however using the methods of reference [27] it is straightforward to write such normal coordinate derivatives at the origin in terms of the corresponding fully-covariant derivatives, denoted by indices trailing the semicolon (;). For example, one can show that 9

$$
\begin{aligned}
D_{\beta_{1}} F_{\beta_{0} \gamma} & \doteq F_{\beta_{0} \gamma ; \beta_{1}} \\
D_{\beta_{2}} D_{\beta_{1}} F_{\beta_{0} \gamma} & \doteq F_{\beta_{0} \gamma ; \beta_{1} \beta_{2}}+\frac{1}{3} F_{\beta_{0} \delta} R_{\beta_{1} \beta_{2} \gamma}^{\delta} \\
D_{\beta_{3}} D_{\beta_{2}} D_{\beta_{1}} F_{\beta_{0} \gamma} & \doteq F_{\beta_{0} \gamma ; \beta_{1} \beta_{2} \beta_{3}}+\frac{1}{2} F_{\beta_{0} \delta} R_{\beta_{1} \beta_{2} \gamma ; \beta_{3}}^{\delta}+F_{\beta_{0} \delta ; \beta_{1}} R_{\beta_{2} \beta_{3} \gamma}^{\delta} \\
D_{\beta_{4}} D_{\beta_{3}} D_{\beta_{2}} D_{\beta_{1}} F_{\beta_{0} \gamma} & \doteq F_{\beta_{0} \gamma ; \beta_{1} \beta_{2} \beta_{3} \beta_{4}}+\frac{3}{5} F_{\beta_{0} \delta} R_{\beta_{1} \beta_{2} \gamma ; \beta_{3} \beta_{4}}^{\delta}+2 F_{\beta_{0} \delta ; \beta_{1}} R_{\beta_{2} \beta_{3} \gamma ; \beta_{4}}^{\delta} \\
& +2 F_{\beta_{0} \delta ; \beta_{1} \beta_{2}} R_{\beta_{3} \beta_{4} \gamma}^{\delta}+\frac{1}{5} F_{\beta_{0} \epsilon} R_{\beta_{1} \beta_{2} \delta}^{\epsilon} R_{\beta_{3} \beta_{4} \gamma}^{\delta}
\end{aligned}
$$

where $\doteq$ indicates equality at the origin only after symmetrization of the $\beta_{i}$ indices. Substitution of equations (B2) into eq. (5b) yields the fully covariant normal coordinate expansion to fifth-order in the normal coordinates,

$$
\begin{aligned}
A_{\gamma}(\phi+\pi(\xi))= & \frac{1}{2}\left\{F_{\beta \gamma}\right\} \xi^{\beta}+\frac{1}{3}\left\{F_{\beta_{0} \gamma ; \beta_{1}}\right\} \xi^{\beta_{0}} \xi^{\beta_{1}}+\frac{1}{8}\left\{F_{\beta_{0} \gamma ; \beta_{1} \beta_{2}}+\frac{1}{3} F_{\beta_{0} \delta} R_{\beta_{1} \beta_{2} \gamma}^{\delta}\right\} \xi^{\beta_{0}} \xi^{\beta_{1}} \xi^{\beta_{2}} \\
& +\frac{1}{3 ! 5}\left\{F_{\beta_{0} \gamma ; \beta_{1} \beta_{2} \beta_{3}}+\frac{1}{2} F_{\beta_{0} \delta} R_{\beta_{1} \beta_{2} \gamma ; \beta_{3}}^{\delta}+F_{\beta_{0} \delta ; \beta_{1}} R_{\beta_{2} \beta_{3} \gamma}^{\delta}\right\} \xi^{\beta_{0}} \xi^{\beta_{1}} \xi^{\beta_{2}} \xi^{\beta_{3}} \\
& +\frac{1}{4 ! 6}\left\{F_{\beta_{0} \gamma ; \beta_{1} \beta_{2} \beta_{3} \beta_{4}}+\frac{3}{5} F_{\beta_{0} \delta} R_{\beta_{1} \beta_{2} \gamma ; \beta_{3} \beta_{4}}^{\delta}+2 F_{\beta_{0} \delta ; \beta_{1}} R_{\beta_{2} \beta_{3} \gamma ; \beta_{4}}^{\delta}\right. \\
& \left.\quad+2 F_{\beta_{0} \delta ; \beta_{1} \beta_{2}} R_{\beta_{3} \beta_{4} \gamma}^{\delta}+\frac{1}{5} F_{\beta_{0} \epsilon} R_{\beta_{1} \beta_{2} \delta}^{\epsilon} R_{\beta_{3} \beta_{4} \gamma}^{\delta}\right\} \xi^{\beta_{0}} \xi^{\beta_{1}} \xi^{\beta_{2}} \xi^{\beta_{3}} \xi^{\beta_{4}} \\
& +\mathcal{O}\left(\xi^{6}\right) .
\end{aligned}
$$

\footnotetext{
${ }^{2}$ The authors suspect that the fourth derivative of a rank-two tensor implied in reference [27] is not entirely correct. The corresponding coefficients presented here for the field strength, equation $(\overline{B 2 d})$, have been verified independently.
} 
All coefficients in braces $\{\cdots\}$ are evaluated at the origin, where the basis vectors for the normal coordinate system coincide with those of the original system. Since the potential on the left hand side of this equation is not a vector at the origin, its indices must refer to the normal coordinate basis. (This is also true of equations (14b)-(14d)). The results of (B3) agree with those of [2] to order $\mathcal{O}\left(\xi^{3}\right)$. 


\section{REFERENCES}

[1] B. DeWitt, Dynamical Theory of Groups and Fields, (Gordon and Breach, New York 1965).

H.P. McKean and J.M. Singer, J. Diff. Geom. 5, 233 (1971).

R.T. Seeley, Amer. Math. Soc. 10, 288 (1967).

P.B. Gilkey, J. Diff. Geom. 10, 601 (1975).

[2] M. Lüscher, Ann. Phys. 142, 359 (1982).

[3] V.P. Gusynin, Nucl. Phys. B333, 296 (1990).

[4] R. Nepomechie, Phys. Rev. D31, 3291 (1985).

M. Reuter, Phys. Rev. D31, 1374 (1985).

[5] R.B. Mann, D.G.C. McKeon, T. Steele and L. Tarasov, Nucl. Phys. B311, 630 (1989).

[6] L. Culumovic and D.G.C. McKeon, Phys. Rev. D38, 3831 (1988).

[7] D. Fliegner, P. Haberl, M.G. Schmidt and C. Schubert, DESY preprint 94-221, hep-th/9411177.

ibid, hep-th/9505077.

[8] J. Beckenstein and L. Parker, Phys. Rev. D19, 483 (1979).

[9] L. Culumovic, D.G.C. McKeon and T.N. Sherry, Ann. of Phys. 197, 94 (1989).

L. Culumovic and D.G.C. McKeon, Can. J. Phys. 68, 1166 (1990).

D.G.C. McKeon and S.K. Wong, Int. J. Mod. Phys. A10, 2181 (1995).

F.A. Dilkes and D.G.C. McKeon, Phys. Rev. D52, 4668 (1995), hep-th/9502075.

[10] M.J. Strassler, Nucl. Phys. B385, 145 (1992).

A.M. Polyakov, Gauge Fields and Strings section 9.3 (Harwood Academic, Chur 1987).

[11] M.G. Schmidt and C. Schubert, Phys. Lett. B318, 438 (1993), hep-th/9309055.

ibid, hep-ph/9412358.

D. Flieger, M.G. Schmidt and C. Schubert, Z. Phys C64, 111 (1994), hep-ph/9401221.

[12] D.G.C. McKeon, Can. J. Phys. 70, 652 (1992).

D.G.C. McKeon and A. Rebhan, Phys. Rev. D48, 2891 (1993).

[13] D.G.C. McKeon and T.N. Sherry, Mod. Phys. Lett. (in press), Int. J. of Theor. Phys. 32, 1105 (1993).

[14] D.G.C. McKeon and S.K. Wong, J. Math. Phys. 36, 1691 (1995).

[15] D.G.C. McKeon, Ann. of Phys. 224, 139 (1993).

[16] M.G. Schmidt and C. Schubert, Phys. Lett. B331, 69 (1994).

[17] B.S. DeWitt, Rev. Mod. Phys. 29, 377 (1957).

K.S. Chen, J. Math. Phys. 13, 1723 (1972).

J. Dowker, J. Phys. A7, 1256 (1974).

G.A. Ringwood, J. Phys. A9, 1253 (1976).

[18] M.S. Marinov, Phys. Rep. 60, 1 (1980).

D.C. Khandakar, S.V. Lawande and K.V. Bhagwat, Path Integral Methods and their Applications (World Scientific, Singapore 1993).

[19] F. Bastianelli, Nucl. Phys. B376, 113 (1992), hep-th/9112035.

F. Bastianelli and P. Van Nieuwenhuizen, Nucl. Phys. B389, 53 (1993), hep-th/9208059.

[20] L. Parker and D. Toms, Phys. Rev. D31, 953 (1985).

I. Jack and L. Parker, Phys. Rev. D31, 2439 (1985).

[21] R.P. Feynman, Rev. Mod. Phys. 20, 367 (1948). 
[22] A. Fock, Phys. Z. Sowjetunion 12, 404 (1937).

C. Crönstrom, Phys. Lett. 90B, 267 (1980).

M.A. Schifman, Nucl. Phys. B173, 13 (1980).

[23] V.P. Gusynin and I.A. Shovkovy, Preprint UG - 9/95, hep-ph/9509383.

[24] C. Itzykson and J.B. Zuber, Quantum Field Theory (McGraw-Hill, New York, 1980).

[25] L. Parker, in Recent Developments in Gravitation, Cargèse 1978 (ed. M. Levy and S. Deser, Plenum Press, London, 1978).

[26] J. Honerkamp, Nucl. Phys. B36, 130 (1972).

[27] L. Alvarez-Gaumé, D.Z. Freedman and S. Mukhi, Ann. of Phys. 134, 85 (1981).

[28] M. Leblanc, R.B. Mann, D.G.C. McKeon and T.N. Sherry, Nucl. Phys. B349, 494 (1991).

[29] O. Veblen, Invariants of Quadratic Differential Forms (Cambridge Press, Cambridge 1927).

L. Eisenhart, Riemann Geometry (Princeton 1965).

A.Z. Petrov, Einstein Spaces (Pegamon Press, New York, 1969).

[30] D.G.C. McKeon, Can. J. Phys. 67, 837 (1989).

[31] D.G.C. McKeon and T.N. Sherry, Phys. Rev. D35, 3854 (1987).

D.G.C. McKeon, Mod. Phys. Lett. A6, 3711 (1991).

[32] D.J. O'Connor, PhD dissertation (University of Maryland), 1985.

D.J. O'Connor, Nucl. Phys. B298, 429 (1988). 


\section{TABLES}

TABLE I. Possible contributions to the various coefficients of the DeWitt expansion.

\begin{tabular}{ll}
\hline \hline Coefficient & Contributions \\
\hline \hline$a_{0}\left(x_{0}, \Delta\right)$ & 1 \\
\hline$a_{1}\left(x_{0}, \Delta\right)$ & $(\Delta \cdot D)^{k} V,(\Delta \cdot D)^{k}\left(\Delta^{\alpha} D_{\beta} F_{\alpha}^{\beta}\right),(\Delta \cdot D)^{k}\left(\Delta^{\alpha} \Delta^{\beta} F_{\alpha \mu} F_{\beta}{ }^{\mu}\right)$ \\
\hline$a_{2}\left(x_{0}, \Delta\right)$ & $(\Delta \cdot D)^{k}\left(D^{2} V\right),(\Delta \cdot D)^{k} V(\Delta \cdot D)^{l} V$, \\
& $(\Delta \cdot D)^{k} V(\Delta \cdot D)^{l}\left(\Delta^{\alpha} D_{\beta} F_{\alpha}^{\beta}\right),(\Delta \cdot D)^{k}\left(\Delta^{\alpha} D_{\beta} F_{\alpha}{ }^{\beta}\right)(\Delta \cdot D)^{l} V$, \\
& $(\Delta \cdot D)^{k}\left(\Delta^{\alpha} \Delta^{\beta} F_{\alpha \mu} F_{\beta}{ }^{\mu}\right)(\Delta \cdot D)^{l} V,(\Delta \cdot D)^{k} V(\Delta \cdot D)^{l}\left(\Delta^{\alpha} \Delta^{\beta} F_{\alpha \mu} F_{\beta}{ }^{\mu}\right)$, \\
& $(\Delta \cdot D)^{k} F^{\alpha \beta}(\Delta \cdot D)^{l} F_{\alpha \beta}$, \\
& $(\Delta \cdot D)^{k}\left(\Delta^{\alpha} F_{\alpha \mu}\right)(\Delta \cdot D)^{l}\left(D_{\beta} F^{\beta \mu}\right),(\Delta \cdot D)^{k}\left(D_{\beta} F^{\beta \mu}\right)(\Delta \cdot D)^{l}\left(\Delta^{\alpha} F_{\alpha \mu}\right)$, \\
& $(\Delta \cdot D)^{k}\left(\Delta^{\alpha} D_{\beta} F_{\alpha}{ }^{\beta}\right)(\Delta \cdot D)^{l}\left(\Delta^{\alpha} D_{\beta} F_{\alpha}{ }^{\beta}\right)$, \\
& $(\Delta \cdot D)^{k}\left(\Delta^{\alpha} D_{\beta} F_{\alpha}{ }^{\beta}\right)(\Delta \cdot D)^{l}\left(\Delta^{\alpha} \Delta^{\beta} F_{\alpha \mu} F_{\beta}{ }^{\mu}\right)$, \\
& $(\Delta \cdot D)^{k}\left(\Delta^{\alpha} \Delta^{\beta} F_{\alpha \mu} F_{\beta}{ }^{\mu}\right)(\Delta \cdot D)^{l}\left(\Delta^{\alpha} D_{\beta} F_{\alpha}{ }^{\beta}\right)$, \\
& $(\Delta \cdot D)^{k}\left(\Delta^{\alpha} \Delta^{\beta} F_{\alpha \mu} F_{\beta}{ }^{\mu}\right)(\Delta \cdot D)^{l}\left(\Delta^{\alpha} \Delta^{\beta} F_{\alpha \mu} F_{\beta}{ }^{\mu}\right)$ \\
\hline$\vdots$ & etc. \\
\hline \hline
\end{tabular}

(All fields and covariant derivatives are evaluated at $x_{0}$.)

$$
(k, l=0,1,2, \cdots)
$$

